\title{
General, Specific and Unique Cognitive Factors Involved in Anxiety and Depressive Disorders
}

\author{
J. Drost • A. J. W. Van der Does · N. Antypa • \\ F. G. Zitman $\cdot$ R. Van Dyck $\cdot$ Ph. Spinhoven
}

Published online: 19 October 2011

(c) The Author(s) 2011. This article is published with open access at Springerlink.com

\begin{abstract}
Comorbidity among anxiety and depressive disorders is the rule rather than the exception. The Integrative Hierarchical Model proposes that each of these disorders contains general (common to all), specific (common to some) and unique components. However, research into this model is limited and hampered by small (clinical) sample sizes. The aim of the present study is to investigate the incremental validity of the cognitive constructs Anxiety Sensitivity, Pathological Worry and Cognitive Reactivity to sad mood over and above the personality traits neuroticism and extraversion. Symptomatic $(N=1,111)$ and remitted $(N=834)$ patients were selected from the 2,981 participants of the Netherlands Study of Depression and Anxiety (NESDA). Results revealed both specific and unique cognitive components of anxiety and depression. Across symptomatic and remitted groups, Anxiety Sensitivity was specific to social anxiety disorder and panic disorder, Aggression Reactivity was a unique component of dysthymia, and Rumination on Sadness was unique to major depressive disorder. We conclude that cognitive constructs have additional value in understanding anxiety and depressive disorders. Moreover, they
\end{abstract}

J. Drost $(\bowtie) \cdot$ A. J. W. Van der Does · N. Antypa ·

$\mathrm{Ph}$. Spinhoven

Institute of Psychology, Leiden University, Wassenaarseweg 52, 2333 AK Leiden, The Netherlands

e-mail: jdrost@fsw.leidenuniv.nl

A. J. W. Van der Does · F. G. Zitman - Ph. Spinhoven Department of Psychiatry, Leiden University Medical Center, Leiden, The Netherlands

R. Van Dyck

Department of Psychiatry, VU University Medical Center, Amsterdam, The Netherlands prove to be more than mere epiphenomena of current disorders.

Keywords Anxiety · Depression · Cognition · Anxiety sensitivity $\cdot$ Pathological worry $\cdot$ Cognitive reactivity

\section{Introduction}

Anxiety and depressive disorders not only have high prevalences, but also high comorbidity rates (Angst 1996; de Graaf et al. 2002; Kessler et al. 1994; Sartorius et al. 1996). This has resulted in an ongoing debate about the breakdown of disorders as postulated in DSM-IV-TR (APA 2000). There is considerable overlap between anxiety and depressive disorders, which is also expressed in a certain degree of similarity in symptomatology, etiology, vulnerability factors and therapeutic interventions.

Etiological models have followed these developments and introduced common higher order factors accounting for comorbidity. The most promising psychological model explaining the findings reported in research and matching the experiences in clinical practice is the Integrative Hierarchical Model (IHM) for anxiety and depression introduced by Mineka et al. (1998). This model, a revised version of the tripartite model (Clark and Watson 1991), has recently attracted a lot of interest (e.g., Prenoveau et al. 2010). According to this model each disorder contains a general, a specific, and a unique component. The general component refers to a factor that anxiety and depressive disorders have in common, the specific component is shared with certain disorders but not all, and the unique component is an aspect characteristic of a particular disorder differentiating it from all the others. Hence, the model addresses the comorbidity issue while still 
acknowledging the heterogeneity of the disorders (Kotov et al. 2007; Mineka et al. 1998).

In line with its predecessor-the tripartite model-the general component of IHM consists of the personality trait Neuroticism. Elevated scores across anxiety and depressive disorders have been reported numerous times and studies investigating a hierarchical structure within the emotional disorders have confirmed its position as a higher order factor (Norton and Mehta 2007; Norton et al. 2005; Sexton et al. 2003). Extraversion is another influential Big Five personality trait. In both Clark and Watson's tripartite model as well as Barlow's Three Factor Model, extraversion constitutes a component unique to depression. This position was questioned however after several studies demonstrated links with Social Anxiety Disorder (SAD) (e.g., Brown et al. 1998; Norton and Mehta 2007) and to a lesser extent Generalized Anxiety Disorder (GAD) (Norton et al. 2005). While not in line with the original hierarchical models it does fit well within the IHM where it reflects a specific component.

Potential candidates for specific or unique components can be found within the wide array of cognitive constructs which includes factors such as rumination on sadness, selffocused attention, and attentional bias to threat. Some of these constructs, like self-focused attention, were originally linked to one or several disorders but as research progressed have later been established as more general components (Ingram 1990). Others are still awaiting further clarification. Three of these cognitive constructs which, though related to trait anxiety or neuroticism, cannot be totally accounted for by these general traits are anxiety sensitivity (AS), pathological worry (PW) and cognitive reactivity to sad mood (CR) (Cox et al. 1999; Meyer et al. 1990; Reiss et al. 1986; Van der Does 2002). AS has been defined as the fear of anxiety related sensations resulting from beliefs held about their potentially harmful physical, psychological or social consequences (Reiss and McNally 1985; Reiss et al. 1986). It is viewed as a dispositional characteristic which is relatively stable over time (for an overview see p. 68 of R. A. Peterson and Plehn 1999) and has mainly been linked to panic disorder (PD). PW on the other hand is the key feature of GAD (DSM-IV-TR; APA 2000) and can be defined as an unwanted, uncontrollable, aversive cognitive activity associated with negative thoughts and emotional discomfort (Borkovec 1994). It is considered a trait like construct and is thought to share the same underlying cognitive process as rumination (Watkins 2008). Contrary to AS and PW where the main focus is on anxiety disorders, the concept of CR has so far only been investigated in relation to depression. $\mathrm{CR}$ is defined as the extent to which dysfunctional schemas are activated when mood decreases. It is reported to successfully distinguish euthymic individuals with and without a history of depression with the remitted group showing consistently higher reactivity (Merens et al. 2005; Miranda et al. 1998, Moulds et al. 2008; Van der Does 2002, 2005; Williams et al. 2008). Furthermore, high CR has also shown to increase the risk of depressive relapse (Segal et al. 1999, 2006).

Although all three constructs are mainly linked to one specific form of psychopathology which suggests that they are unique factors in the IHM model, there are also studies reporting otherwise. AS for example, has besides its connection to PD also been linked to all the other anxiety disorders as well as depression (Otto et al. 1995; Rector et al. 2007; Rodriguez et al. 2004). Similar findings have been reported for PW (Chelminski and Zimmerman 2003; Starcevic 1995; Starcevic et al. 2007) suggesting its relationship with GAD is not an exclusive one. CR has thus far only been studied in relation to depressive disorders, specifically those with a history of depression, where theoretically its influence is expected to be the most pronounced.

Studies investigating the role of cognitive constructs within hierarchical models are limited. PW has been mainly studied as a measure representing a key characteristic of GAD, but GAD is more encompassing than unwanted, uncontrollable, aversive cognitive activity and this cognitive process may have a wider relevance as a predictor of GAD, but also of related disorders (Watkins 2008). To our knowledge, both PW and CR have thus far not been tested as predictors in a hierarchical model. AS on the other hand has been included in a few such studies, where it differentiated PD from other anxiety and depressive disorders (Norton and Mehta 2007; Norton et al. 2005). However, studies did not investigate the direct contributions of lower order factors of AS and were hampered by either the use of analogue or small clinical samples. Moreover, only a couple of cognitive constructs were used and outcome measures consisted of symptoms, not actual psychiatric diagnoses.

The main aim of the present study is to investigate the incremental validity of the cognitive constructs AS, PW and $\mathrm{CR}$ in predicting specific depressive and anxiety disorders over and above the Big Five personality traits of neuroticism and extraversion. We will investigate this question both in symptomatic patients and in participants who are in remission, hence exploring whether elevated scores on cognitive constructs merely reflect an epiphenomenon of current psychopathology or not. We will address several limitations from previous studies by using both clinical and healthy samples. Moreover, the availability of a very large sample permits us to investigate multiple personality traits and cognitive constructs simultaneously in order to investigate their relative predictive power, while also taking comorbidity into account. The 
sample comes from diverse settings (community, primary care, and specialised mental health care) and includes patients in various stages of illness, enhancing generalizability.

\section{Method}

\section{Participants and Setting}

Participants were selected from the Netherlands Study of Depression and Anxiety (NESDA), an ongoing 8-year multi-site naturalistic, longitudinal cohort study including 2,981 adult subjects aged 18 through 65 years. The sample consists of 687 healthy controls, 2,294 persons with a lifetime diagnosis of depression or anxiety disorder of which 1,342 have a current diagnosis (past month). In order to be representative of those with depressive and anxiety disorders respondents in different stages of the developmental history of the disorders (normal, high familial risk, subthreshold disorders, first and recurrent episodes) and from different health care settings (community, primary care and specialized mental health care) were included. The NESDA community sample had been previously identified in two population based studies: the Netherlands Mental Health Survey and Incidence Study (NEMESIS; Bijl et al. 1998) and the Adolescents at Risk of Anxiety and Depression (ARIADNE) study (Landman-Peeters et al. 2005). The recruitment of primary care patients took place through 65 general practitioners-using a three-stage screening method-and the recruitment of psychiatric outpatients through 17 mental health care institutions.

Across recruitment settings uniform in and exclusion criteria were used. A general inclusion criterion was an age of 18 through 65 years. An exclusion criterion was a primary psychotic, obsessive compulsive, bipolar or severe addiction disorder. In addition patients who were not fluent in Dutch were excluded. A more extensive description of the rationale, method and recruitment strategy can be found elsewhere (Penninx et al. 2008).

In the current study the psychological disorders of interest are anxiety disorders SAD, GAD, PD (with or without agoraphobia), and depressive disorders dysthymia (DD) and Major Depressive Disorder (MDD). Two psychiatric groups will be investigated; patients with a diagnosis at time of assessment (current diagnosis) and patients who fulfilled criteria of the disorder of interest at some point in life but did not meet full DSM-IV criteria in the past month (remission). Using a current sample dovetails nicely with existing literature allowing results to be easily compared. The remission sample provides an opportunity to replicate the findings of the current sample and to challenge the possibility of results merely reflecting epiphenomena of current disorders. This was further safeguarded by controlling for residual symptoms.

\section{Measures}

Within the scope of NESDA many different measures have been administered. Only the ones relevant to the present study will be discussed here.

\section{Assessment of Psychiatric Diagnoses}

Depressive disorders (DD, MDD) and anxiety disorders (SAD, GAD, PD) were diagnosed using the Composite Interview Diagnostic Instrument (CIDI-WHO lifetime version 2.1; Ter Smitten et al. 1998). The CIDI is a worldwide used instrument which classifies diagnoses according to DSM-IV criteria (APA 1994). It has shown high interrater reliability (Wittchen et al. 1991), high testretest reliability (Wacker et al. 2006) and high validity for depressive and anxiety disorders (Farmer et al. 1987; Wittchen 1994; Wittchen et al. 1989). The CIDI was conducted by specially trained clinical research staff.

\section{Assessment of General Factors}

Neuroticism The personality trait neuroticism was measured using the NEO Five-Factor Inventory (NEO-FFI) personality questionnaire (Costa and Mccrae 1995). This 60-item questionnaire contains a subscale for Neuroticism consisting of 12-items which are scored on a five-point scale ranging from 'strongly agree' to 'strongly disagree'. Scores ranged from 1 to 5 for each item, and the total score for each domain ranged from a minimum of 12 to a maximum of 60 . The psychometric characteristics are satisfactory (Costa and Mccrae 1995). In the present study internal consistency was satisfactory with $\alpha=0.75$.

\section{Assessment of Specific Factors}

Extraversion The personality trait extraversion was measured in the same manner as neuroticism using a subscale of the NEO-FFI personality questionnaire. Internal consistency in the present study reached an adequate level with $\alpha=0.78$.

Anxiety Sensitivity Anxiety sensitivity was assessed using the Anxiety Sensitivity Index -16 items (Peterson and Reiss 1992; Reiss et al. 1986). This self-report questionnaire indicates the degree to which respondents are concerned about possible negative consequences of anxietyrelated sensations. Items are scored on a 5-point Likert scale ranging from ' $0=$ very little' to ' $4=$ very much'. Total scores range from 0 to 64 . The ASI has high levels of 
internal consistency, good test-retest reliability, and good validity (R. A. Peterson and Plehn 1999; Reiss et al. 1986). Evidence from previous studies concerning the lower-order factorial structure of the ASI was nonconclusive (Cox et al. 1996; Schmidt and Joiner 2002; Vujanovic et al. 2007; Zinbarg et al. 1997). Consequently, the NESDA data were screened beforehand to determine the best fitting factor structure. Considering the large sample size of NESDA this is expected to prove a reliable method. Exploratory factor analyses presented 4 factors with eigenvalues greater than 1, of which 2 showed relatively poor internal consistency. This is in line with previous findings by Vujanovic et al. (2007) who also reported low internal consistency for 2 of the 4 factors. To maintain good internal consistency factors were combined to form 2 factors: a physical concerns factor (ASI-phc) and a social-cognitive concerns factor (ASI-scc). This was based both on theoretical and on statistical grounds. Theoretically it was most logical to combine factors of which the content was related. This theoretical solution matched the statistically emerging solution of a factor analysis with a forced 2 factor solution. The two factors explained $54.6 \%$ of the total variance in ASI scores. Items 7 and 13 were left out as both items showed very low loadings on each of the 2 factors and reliability analyses revealed that removal would improve the internal consistency. Removal of these two items did not affect the factor structure. The internal consistency of the 2 factors used in this study are adequate with $\alpha=0.89$ for the physical concerns factor (items 3, 4, 6, 8, 9, 10, 11, 14) and $\alpha=0.80$ for the social-cognitive concerns factor (items 1, 2, 5, 12, 15, 16). The subdivision into two factors makes AS a candidate for a unique as well as a specific factor.

\section{Assessment of Unique Factors}

Pathological Worry Pathological worry was operationalized using the Penn State Worry Questionnaire (PSWQ; Meyer et al. 1990). Items consist of statements related to worry, each rated on a 5-point Likert-scale ranging from ' $1=$ not at all typical of me' to ' $5=$ very typical of me'. The PSWQ consists of two subscales: a 'General worry' subscale (11 items) and a 'Not-worry' subscale (5 items) (van Rijsoort et al. 1999). The 'General worry' subscale is the strongest of the two (Brown et al. 1992; Meyer et al. 1990; van Rijsoort et al. 1999) and only this subscale was administered in the NESDA study. Psychometric properties of this Dutch 11-item version are not available but the original PSWQ has been proven to be a valid measure of trait worrying unaffected by the content of the worry (Davey 1993; Molina and Borkovec 1994) with high internal consistency, good test-retest reliability and unaffected by social desirability (Meyer et al. 1990).
The adjustments made to the original PSWQ are not expected to have had a negative effect on these characteristics. Internal consistency in the present study was high, namely $\alpha=0.96$.

Cognitive Reactivity to Sad Mood Cognitive reactivity to sad mood was measured using the revised version of the Leiden Index of Depression Sensitivity (LEIDS-R; Van der Does 2002; Williams et al. 2008). The LEIDS-R is a selfreport instrument which has been found to reliably discriminate between never-depressed and recovered depressed groups (e.g., Firk and Markus 2009; Merens et al. 2005; Moulds et al. 2008; Van der Does 2002). LEIDS-R scores also correlate with biological vulnerability markers of depression: response to acute tryptophan depletion (Booij and Van der Does 2007) and a serotonin transporter gene polymorphism (Antypa et al. 2010).

The LEIDS-R consists of 34 items divided over six subscales: Hopelessness/Suicidality Reactivity (HOP), Acceptance/Coping (ACC), Aggression Reactivity (AGG), Control/Perfectionism (CTR), Risk Avoidance (RAV) and Rumination on Sadness (RUM). Participants are asked to indicate whether and how their thinking patterns change when they experience mild dysphoria by scoring each item on a 5-point Likert-scale ranging from 0 'not at all' to 4 'very strongly' applicable to me. In the present sample internal consistencies of the subscales ranged from $\alpha=0.62$ (ACC) to $\alpha=0.86$ (HOP).

\section{Covariates}

Assessment of Demographic and Personal Characteristics

Detailed sociodemographic and socioeconomic data were collected, of which sex, age and number of years of education, were used in the present study.

\section{Assessment of Symptom Levels}

In order to control for residual symptoms in the remission sample, two broad and well established measures of anxiety and depressive symptomatology were used:

Anxiety The 21-item Beck Anxiety Inventory (BAI) (Beck et al. 1988) provides a reliable and valid assessment of anxiety symptomatology. In previous research it has shown high internal consistency, high test-retest reliability and good concurrent and discriminant validity (Ferguson 2000). Internal consistency in the present study was high with $\alpha=0.95$.

Depression Depressive symptoms were measured using the Inventory of Depressive Symptomatology (IDS) a 
30-item self report questionnaire. Psychometric properties are satisfactory (Rush et al. 1996). In the present study internal consistency was satisfactory with $\alpha=0.91$.

\section{Procedure}

The study protocol was approved centrally by the Ethical Review Board of the VU University Medical Centre and subsequently by local review boards of each participating centre. NESDA subjects were assessed during a 4-h clinic visit at one of the seven field centre locations.

Once full verbal and written information about the study was given, written informed consent was obtained from all participants of the baseline assessment. Interviews were administered with computer-assisted personalized interviewing (CAPI) procedures with data entry checks on outliers and routing. When necessary the interview was taken in more than one session to avoid fatigue. Specially trained research assistants conducted the assessments. All interviews were taped to monitor data-quality and interviewer performance. In addition, the data monitoring centre routinely carried out data quality checks to review missing data and check for inconsistencies. After completion of the assessment respondents were compensated with a small incentive (gift certificate of 15 euros and payment of travel costs) for their time and cooperation.

\section{Statistical Analyses}

First scores on all measures were compared between participants meeting criteria for current disorder and participants who did not meet these criteria, as well as comparing participants with a disorder in remission to a control group with no lifetime disorders.

The ability of measures to (independently) predict an anxiety or depressive diagnosis was investigated within the total sample of participants who fulfilled criteria for a current anxiety or depressive disorder as well as in a group with a disorder in remission (cp. Gibb et al. 2007). A benefit of this analysis is that it includes a built-in psychiatric control group. Binominal logistic regression was conducted to assess this relative specificity of cognitive constructs using a hierarchical procedure. Demographics (gender, age and number of years of education) were entered as covariates by using forced entry into the model. In order to test whether the cognitive constructs would uphold their predictive value after correcting for neuroticism and extraversion, personality traits were also entered into the model using forced entry. The independent variables of interest, namely PSWQ, ASI-phc, ASI-scc and the six LEIDS-R-subscales were entered last, using a stepwise backward procedure (likelihood ratio) with a removal probability of 0.01 . A stepwise backward procedure was chosen over a stepwise forward procedure because the latter has a higher risk of Type II error due to suppressor effects (Field 2005).

Analyses were conducted for both the current diagnosis and diagnosis in remission group, with the only difference being that in the remission models BAI and IDS were added as covariates to control for residual symptoms.

A significance level of 0.01 was applied in order to control the familywise error rate and reduce the chances of a Type I error. To guard against multicollinearity the VIF score for each variable in each model was examined. No VIF statistic for any variable was found with a value above 2.7 (tolerance not below 0.37), suggesting that multicollinearity was not a problem for these regression models (Menard 1995; Myers 1990). All analyses were conducted using PASW Statistics package 17.0 (SPSS, INC, Chicago, Illinois, 2009).

\section{Results}

\section{Sample Description}

Demographic information on the sample and detailed information on the personality traits and cognitive constructs can be found in Tables 1 and 2. The NESDA sample consists of 2,981 adult subjects aged between 18 and 65 years, of which complete data including NEO-FFI, PSWQ, ASI and LEIDS-R scores were available for 2,590 participants $(86.9 \%)$. The study sample mean age is 42.3 years $(\mathrm{SD}=13.1)$, and $67.2 \%$ is female. Almost half $(43.2 \%)$ of the participants has one or more current anxiety or depressive disorders and three-quarters $(75.6 \%)$ has a lifetime anxiety or depressive disorder.

\section{Comparison of Healthy Controls with Acute} and Remitted Diagnostic Groups

When comparing participants with a current disorder $(N=1,111)$ to the group without current psychopathology $(N=1,462)$ the former group scores significantly higher $(P<0.001)$ on the personality traits (except for extraversion where the relationship is reversed) and all cognitive constructs. Cohen's $d$ effect size is 0.33 (small effect) for LEIDS-R-acc and all others vary between 0.56 and 1.37 (large effect).

With regard to demographics, a significant difference $(P<0.001)$ for education was found, with the current disorder group completing fewer years of education $(d=0.27)$. Differences in gender and age were not significant.

Similar results were found when comparing participants with a disorder in remission $(N=834)$ to participants who had never experienced psychopathology in their lives 
Table 1 Comparison of participants with a current disorder and those without current psychopathology

\begin{tabular}{|c|c|c|c|c|c|c|}
\hline Current diagnosis & $\begin{array}{l}\text { No current disorder } \\
(N=1,462)\end{array}$ & $\begin{array}{l}\text { SAD } \\
(N=462)^{\mathrm{a}}\end{array}$ & $\begin{array}{l}\text { GAD } \\
(N=322)^{\mathrm{a}}\end{array}$ & $\begin{array}{l}\mathrm{PD} \\
(N=422)^{\mathrm{a}}\end{array}$ & $\begin{array}{l}\mathrm{DD} \\
(N=228)^{\mathrm{a}}\end{array}$ & $\begin{array}{l}\text { MDD } \\
(N=647)^{\mathrm{a}}\end{array}$ \\
\hline Gender: female & $67.4 \%$ & $66.0 \%$ & $64.3 \%$ & $69.9 \%$ & $64.0 \%$ & $66.2 \%$ \\
\hline Age & $42.5(13.7)$ & $41.7(11.9)$ & 42.7 (11.9) & $42.2(12.0)$ & $44.4(11.5)$ & $42.3(12.2)$ \\
\hline Education (years) & $12.7(3.2)$ & $11.7(3.3)$ & $11.6(3.3)$ & $11.4(3.3)$ & $11.5(3.5)$ & $11.6(3.1)$ \\
\hline Neuroticism & $31.3(8.4)$ & $43.8(6.6)$ & $44.1(6.5)$ & $41.6(7.3)$ & $44.5(6.0)$ & $43.1(6.6)$ \\
\hline Extraversion & $39.8(6.7)$ & $31.7(6.4)$ & $32.3(6.2)$ & $34.4(7.0)$ & $30.5(6.9)$ & $32.1(6.6)$ \\
\hline PSWQ & $25.2(10.4)$ & $39.2(9.6)$ & $42.0(8.8)$ & $38.3(10.0)$ & $41.5(8.5)$ & $39.5(9.7)$ \\
\hline ASI-phc & $4.6(4.7)$ & $9.5(6.8)$ & $9.7(6.6)$ & $12.0(7.0)$ & $9.4(6.5)$ & $8.6(6.5)$ \\
\hline ASI-scc & $4.8(3.1)$ & $9.6(4.5)$ & $9.2(4.7)$ & $9.3(4.9)$ & $9.5(4.8)$ & $8.7(4.7)$ \\
\hline LEIDS-R-rum & $7.0(4.7)$ & $12.3(4.6)$ & $12.5(4.8)$ & $11.3(5.0)$ & $13.3(4.2)$ & $12.6(4.5)$ \\
\hline LEIDS-R-hop & $2.9(3.2)$ & $7.9(5.1)$ & $8.1(5.4)$ & $6.9(5.2)$ & $9.2(5.1)$ & $8.2(5.2)$ \\
\hline LEIDS-R-acc & $1.2(1.8)$ & $2.0(2.3)$ & $2.1(2.4)$ & $2.0(2.5)$ & $2.0(2.4)$ & $2.0(2.4)$ \\
\hline LEIDS-R-agg & $3.5(3.4)$ & $7.0(5.1)$ & $6.7(4.8)$ & $6.2(5.2)$ & $7.8(5.1)$ & $6.7(4.9)$ \\
\hline LEIDS-R-ctr & $4.6(3.7)$ & $7.2(3.8)$ & $7.0(3.9)$ & $6.8(4.1)$ & $7.2(3.8)$ & $6.9(4.0)$ \\
\hline LEIDS-R-rav & $6.4(4.4)$ & $11.8(4.5)$ & $11.5(4.6)$ & $10.7(4.9)$ & $12.1(4.5)$ & $11.4(4.5)$ \\
\hline $\mathrm{N}$ current disorders & 0.0 & $2.3(1.2)$ & $2.7(1.1)$ & $2.3(1.2)$ & $3.0(1.1)$ & $2.2(1.1)$ \\
\hline
\end{tabular}

${ }^{a}$ Due to comorbidity participants in the disorder columns might overlap

Table 2 Comparison of participants who are in remission and those without any psychiatric history

\begin{tabular}{|c|c|c|c|c|c|c|}
\hline $\begin{array}{l}\text { Diagnosis in } \\
\text { remission }\end{array}$ & $\begin{array}{l}\text { No psychiatric history } \\
(N=628)\end{array}$ & $\begin{array}{l}\text { SAD } \\
(N=192)^{\mathrm{a}}\end{array}$ & $\begin{array}{l}\mathrm{GAD} \\
(N=194)^{\mathrm{a}}\end{array}$ & $\begin{array}{l}\mathrm{PD} \\
(N=188)^{\mathrm{a}}\end{array}$ & $\begin{array}{l}\mathrm{DD} \\
(N=157)^{\mathrm{a}}\end{array}$ & $\begin{array}{l}\text { MDD } \\
(N=694)^{\mathrm{a}}\end{array}$ \\
\hline Gender: female & $62.3 \%$ & $69.8 \%$ & $72.7 \%$ & $77.1 \%$ & $74.5 \%$ & $71.9 \%$ \\
\hline Age & $41.5(14.6)$ & $43.7(12.5)$ & $43.4(12.5)$ & $40.4(12.4)$ & $46.6(11.6)$ & $43.2(12.5)$ \\
\hline Education (years) & $12.8(3.2)$ & $12.4(3.3)$ & $12.5(3.2)$ & $12.4(3.2)$ & $12.2(3.7)$ & $12.6(3.2)$ \\
\hline Neuroticism & $27.1(7.4)$ & $35.7(8.2)$ & $36.5(7.7)$ & $35.9(7.5)$ & $36.0(7.3)$ & $34.8(7.8)$ \\
\hline Extraversion & $42.0(6.2)$ & $36.7(6.5)$ & $37.4(6.3)$ & $38.4(6.7)$ & $36.4(6.7)$ & $37.9(6.7)$ \\
\hline PSWQ & $20.7(8.8)$ & $28.7(10.1)$ & $31.5(10.4)$ & $29.6(10.0)$ & $31.2(10.3)$ & $29.0(10.2)$ \\
\hline ASI-phc & $3.3(3.8)$ & $6.2(5.5)$ & $6.1(5.0)$ & $7.6(5.9)$ & $6.0(5.1)$ & $5.4(5.2)$ \\
\hline ASI-scc & $3.8(2.4)$ & $6.3(3.5)$ & $6.0(3.5)$ & $6.1(3.7)$ & $6.0(3.4)$ & $5.5(3.3)$ \\
\hline LEIDS-R-rum & $4.8(4.0)$ & $9.1(4.8)$ & $9.3(4.7)$ & $9.0(4.8)$ & $9.6(4.4)$ & $8.9(4.5)$ \\
\hline LEIDS-R-hop & $1.6(2.3)$ & $4.5(3.9)$ & $4.4(3.7)$ & $4.2(3.8)$ & $4.8(4.0)$ & $4.1(3.6)$ \\
\hline LEIDS-R-acc & $0.9(1.6)$ & $1.4(1.9)$ & $1.6(2.2)$ & $1.5(1.9)$ & $1.8(2.4)$ & $1.5(2.1)$ \\
\hline LEIDS-R-agg & $2.6(2.7)$ & $4.7(4.0)$ & $4.7(3.6)$ & $3.8(3.3)$ & $5.1(3.9)$ & $4.4(3.7)$ \\
\hline LEIDS-R-ctr & $3.4(3.1)$ & $5.5(3.8)$ & $6.3(4.2)$ & $5.3(3.3)$ & $6.3(4.0)$ & $5.6(3.9)$ \\
\hline LEIDS-R-rav & $4.4(3.6)$ & $8.5(4.3)$ & $8.9(4.4)$ & $8.3(4.5)$ & $8.7(4.1)$ & $8.2(4.3)$ \\
\hline $\mathrm{N}$ disorders in remission & 0.0 & $2.4(1.1)$ & $2.6(1.0)$ & $2.4(1.0)$ & $2.8(0.9)$ & $1.8(0.9)$ \\
\hline
\end{tabular}

Participants in remission = History of psychopathology, but none of the current diagnoses

${ }^{a}$ Due to comorbidity participants in the disorder columns might overlap

$(N=628)$, with highly significant $(P<0.001)$ differences for all personality traits and cognitive constructs. LEIDSR-acc had a Cohen's $d$ effect size of 0.29 (small effect) and all others varied between 0.50 and 0.96 (large effect). Results were all in the expected direction.

In demographics, a significant difference was observed in the gender distribution $(\varphi=-0.10)$ indicating that the remission group contained a higher percentage of females. Further, there was a negligible effect $(d=0.13, P=0.02)$ for age reflecting a slightly older remission sample. No difference was found for number of years of education. Demographic variables were included as covariates in all analyses, and between group differences in demographics were thereby controlled for. 
Hierarchical Regression Analyses Investigating Incremental Validity

Binomial logistic regression was conducted for each of the disorders. Logistic coefficients, Wald statistic, odds ratio and $95 \%$ confidence intervals from each logistic regression analysis are presented in Tables 3, 4, 5 and 6. As shown above, psychopathology groups scored significantly higher than healthy controls (with the exception of extraversion where-as expected-an inverse relation was reported). Because the present analysis is conducted in participants with an acute disorder or a disorder in remission only (excluding healthy controls), associations in an unexpected direction merely reflect that the predictor is not so important for that particular disorder in comparison to the other disorders it is compared to. These associations indicate that the predictor is less dominant in a particular disorder and does not imply that the predictor constitutes a protective factor for that disorder. Therefore below only predictors with a significant contribution to the model in the expected direction will be discussed.

Table 3 Prediction of current anxiety disorders by personality factors and cognitive constructs, controlled for demographics

\begin{tabular}{|c|c|c|c|c|c|c|c|c|c|c|c|c|}
\hline & \multicolumn{4}{|l|}{ SAD } & \multicolumn{4}{|l|}{ GAD } & \multicolumn{4}{|l|}{ PD } \\
\hline & $B$ & Wald test & OR & $95 \% \mathrm{CI}$ & $B$ & Wald test & OR & $95 \% \mathrm{CI}$ & $B$ & Wald test & OR & $95 \% \mathrm{CI}$ \\
\hline \multicolumn{13}{|l|}{ Forced entry } \\
\hline Sex & 0.12 & 0.57 & 1.11 & $0.85-1.46$ & 0.26 & 2.99 & 1.30 & $0.97-1.74$ & -0.21 & 1.95 & 0.82 & $0.61-1.09$ \\
\hline Age & -0.01 & 1.53 & 0.99 & $0.98-1.00$ & 0.01 & 2.76 & 1.01 & $1.00-1.02$ & -0.001 & 0.03 & 1.00 & $0.99-1.01$ \\
\hline Education & -0.01 & 0.17 & 0.99 & $0.95-1.03$ & -0.03 & 1.31 & 0.98 & $0.94-1.02$ & -0.06 & $6.85 * *$ & 0.95 & $0.91-0.99$ \\
\hline Neuroticism & 0.06 & $18.70 * * *$ & 1.06 & $1.03-1.09$ & 0.04 & $7.87 * *$ & 1.04 & $1.01-1.07$ & -0.02 & 2.90 & 0.98 & $0.96-1.00$ \\
\hline Extraversion & -0.04 & $12.87 * * *$ & 0.96 & $0.94-0.98$ & 0.00 & 0.04 & 1.00 & $0.98-1.03$ & 0.03 & $6.43 *$ & 1.03 & $1.01-1.06$ \\
\hline \multicolumn{13}{|c|}{ Stepwise backward } \\
\hline PSWQ & -0.03 & $8.54 * *$ & 0.98 & $0.96-0.99$ & 0.05 & $32.23 * * *$ & 1.05 & $1.03-1.07$ & & & & \\
\hline ASI-phc & & & & & & & & & 0.11 & $108.44 * * *$ & 1.12 & $1.10-1.14$ \\
\hline ASI-scc & 0.08 & $25.16^{* * *}$ & 1.08 & $1.05-1.12$ & & & & & & & & \\
\hline
\end{tabular}

SAD: $R^{2}=0.13$ (Nagelkerke). Model $\chi^{2}(7)=108.66, P<0.001 ;$ GAD: $R^{2}=0.12$ (Nagelkerke). Model $\chi^{2}(6)=94.40, P<0.001 ;$ PD: $R^{2}=0.18$ (Nagelkerke). Model $\chi^{2}(6)=156.03, P<0.001$

In the stepwise backward section of the model, non-significant cognitive factors are not reported

$* P<0.05$; ** $P<0.01$; *** $P<0.001$

Table 4 Prediction of current depressive disorders by personality factors and cognitive constructs, controlled for demographics

\begin{tabular}{|c|c|c|c|c|c|c|c|c|}
\hline & \multicolumn{4}{|l|}{$\mathrm{DD}$} & \multicolumn{4}{|l|}{ MDD } \\
\hline & $B$ & Wald test & OR & $95 \% \mathrm{CI}$ & $B$ & Wald test & OR & $95 \% \mathrm{CI}$ \\
\hline \multicolumn{9}{|l|}{ Forced entry } \\
\hline Sex & -0.02 & 0.02 & 0.98 & $0.70-1.37$ & -0.04 & 0.08 & 0.96 & $0.73-1.27$ \\
\hline Age & 0.03 & $13.88 * * *$ & 1.03 & $1.01-1.04$ & 0.002 & 0.09 & 1.00 & $0.99-1.01$ \\
\hline Education & -0.01 & 0.23 & 0.99 & $0.94-1.04$ & -0.06 & $9.53 * *$ & 0.94 & $0.90-0.98$ \\
\hline Neuroticism & 0.05 & $9.76 * *$ & 1.05 & $1.02-1.08$ & 0.01 & 0.48 & 1.01 & $0.98-1.03$ \\
\hline Extraversion & -0.05 & $14.29 * * *$ & 0.95 & $0.92-0.98$ & -0.04 & $10.74 * *$ & 0.96 & $0.94-0.99$ \\
\hline \multicolumn{9}{|c|}{ Stepwise backward } \\
\hline ASI-phc & & & & & -0.04 & $19.16 * * *$ & 0.96 & $0.94-0.98$ \\
\hline LEIDS-R-rum & & & & & 0.07 & $15.02 * * *$ & 1.07 & $1.03-1.11$ \\
\hline LEIDS-R-hop & & & & & 0.06 & $10.68 * *$ & 1.06 & $1.02-1.10$ \\
\hline LEIDS-R-agg & 0.06 & $11.91 * *$ & 1.06 & $1.03-1.09$ & & & & \\
\hline
\end{tabular}

DD: $R^{2}=0.13$ (Nagelkerke). Model $\chi^{2}(6)=91.79, P<0.001$; MDD: $R^{2}=0.15$ (Nagelkerke). Model $\chi^{2}(8)=133.93, P<0.001$

In the stepwise backward section of the model, non-significant cognitive factors are not reported

$* P<0.05$; ** $P<0.01$; *** $P<0.001$ 
Table 5 Prediction of anxiety disorders in remission by personality factors and cognitive constructs, controlled for demographics, BAI and IDS

\begin{tabular}{|c|c|c|c|c|c|c|c|c|c|c|c|c|}
\hline & \multicolumn{4}{|l|}{ SAD } & \multicolumn{4}{|l|}{ GAD } & \multicolumn{4}{|l|}{ PD } \\
\hline & $B$ & Wald test & OR & $95 \% \mathrm{CI}$ & $B$ & Wald test & OR & $95 \% \mathrm{CI}$ & $B$ & Wald test & OR & $95 \% \mathrm{CI}$ \\
\hline \multicolumn{13}{|l|}{ Forced entry } \\
\hline Sex & 0.01 & 0.004 & 1.01 & $0.70-1.46$ & -0.02 & 0.01 & 0.98 & $0.68-1.42$ & -0.13 & 0.42 & 0.88 & $0.58-1.31$ \\
\hline Age & -0.001 & 0.01 & 1.00 & $0.99-1.01$ & 0.01 & 1.79 & 1.01 & $1.00-1.02$ & -0.03 & $15.56 * * *$ & 0.97 & $0.96-0.99$ \\
\hline Education & -0.01 & 0.19 & 0.99 & $0.94-1.04$ & 0.002 & 0.01 & 1.00 & $0.95-1.06$ & -0.01 & 0.11 & 0.99 & $0.94-1.05$ \\
\hline BAI & -0.004 & 0.07 & 1.00 & $0.96-1.03$ & 0.01 & 0.66 & 1.01 & $0.98-1.05$ & 0.04 & $3.93 *$ & 1.04 & $1.00-1.07$ \\
\hline IDS & -0.003 & 0.05 & 1.00 & $0.97-1.03$ & -0.03 & 3.56 & 0.97 & $0.95-1.00$ & -0.01 & 0.75 & 0.99 & $0.96-1.02$ \\
\hline Neuroticism & 0.03 & 2.98 & 1.03 & $1.00-1.07$ & 0.06 & $16.82 * * *$ & 1.07 & $1.03-1.10$ & 0.02 & 1.23 & 1.02 & $0.99-1.05$ \\
\hline Extraversion & -0.04 & $6.78 * *$ & 0.96 & $0.93-0.99$ & 0.001 & 0.01 & 1.00 & $0.97-1.03$ & 0.01 & 0.69 & 1.01 & $0.98-1.05$ \\
\hline \multicolumn{13}{|c|}{ Stepwise backward } \\
\hline PSWQ & -0.04 & $9.61 * *$ & 0.96 & $0.94-0.99$ & & & & & & & & \\
\hline ASI-phc & & & & & & & & & 0.10 & $28.99 * * *$ & 1.10 & $1.06-1.14$ \\
\hline ASI-scc & 0.11 & $15.91 * * *$ & 1.12 & $1.06-1.18$ & & & & & & & & \\
\hline LEIDS-R-agg & & & & & & & & & -0.10 & $11.95 * * *$ & 0.91 & $0.86-0.96$ \\
\hline
\end{tabular}

SAD: $R^{2}=0.06$ (Nagelkerke). Model $\chi^{2}(9)=35.04, P<=0.001$; GAD: $R^{2}=0.04$ (Nagelkerke). Model $\chi^{2}(7)=23.53, P=0.001$; PD: $R^{2}=0.13$ (Nagelkerke). Model $\chi^{2}(9)=74.18, P<0.001$

In the stepwise backward section of the model, non-significant cognitive factors are not reported

$* P<0.05$; ** $P<0.01$; *** $P<0.001$

Table 6 Prediction of depressive disorders in remission by personality factors and cognitive constructs, controlled for demographics, BAI and IDS

\begin{tabular}{|c|c|c|c|c|c|c|c|c|}
\hline & \multicolumn{4}{|l|}{$\mathrm{DD}$} & \multicolumn{4}{|l|}{ MDD } \\
\hline & $B$ & Wald test & OR & $95 \% \mathrm{CI}$ & $B$ & Wald test & OR & $95 \% \mathrm{CI}$ \\
\hline \multicolumn{9}{|l|}{ Forced entry } \\
\hline Sex & -0.38 & 3.18 & 0.68 & $0.45-1.04$ & -0.23 & 1.13 & 0.80 & $0.53-1.21$ \\
\hline Age & 0.03 & $14.25 * * *$ & 1.03 & $1.02-1.05$ & 0.01 & 0.61 & 1.01 & $0.99-1.02$ \\
\hline Education & -0.02 & 0.63 & 0.98 & $0.92-1.03$ & 0.01 & 0.07 & 1.01 & $0.95-1.07$ \\
\hline BAI & -0.01 & 0.38 & 0.99 & $0.96-1.02$ & -0.01 & 0.09 & 0.99 & $0.95-1.04$ \\
\hline IDS & 0.02 & 1.69 & 1.02 & $0.99-1.05$ & 0.03 & 2.35 & 1.03 & $0.99-1.06$ \\
\hline Neuroticism & 0.01 & 0.21 & 1.01 & $0.98-1.04$ & 0.01 & 0.10 & 1.01 & $0.97-1.04$ \\
\hline Extraversion & -0.03 & 3.62 & 0.97 & $0.94-1.00$ & -0.01 & 0.33 & 0.99 & $0.96-1.03$ \\
\hline \multicolumn{9}{|c|}{ Stepwise backward } \\
\hline ASI-phc & & & & & -0.06 & $10.47 * *$ & 0.94 & $0.90-0.98$ \\
\hline LEIDS-R-rum & & & & & 0.09 & $11.21 * * *$ & 1.09 & $1.04-1.15$ \\
\hline LEIDS-R-agg & 0.08 & $9.44 * *$ & 1.08 & $1.03-1.13$ & & & & \\
\hline
\end{tabular}

DD: $R^{2}=0.08$ (Nagelkerke). Model $\chi^{2}(8)=44.70, P<0.001$; MDD: $R^{2}=0.08$ (Nagelkerke). Model $\chi^{2}(9)=38.04, P<0.001$

In the stepwise backward section of the model, non-significant cognitive factors are not reported

$* P<0.05$; ** $P<0.01$; *** $P<0.001$

\section{Prediction of Current Diagnosis by Cognitive Constructs After Correction for Personality Traits}

In the first series of analyses only participants with a current diagnosis were included $(N=1,111)$. Results of the binomial logistic regressions are presented in Tables 3 and 4. These tables also show model fit and explained variance (Nagelkerke $R^{2}$ ) for each model.
After correction for demographics and personality traits results reveal PSWQ as a predictor of GAD, ASI-physical concerns of PD and ASI-social-cognitive concerns of SAD. Hence, indicating that these cognitive constructs possess additional predictive value over and above neuroticism and extraversion. Inspection of the LEIDS-Rsubscales reveals that the hopelessness subscale (LEIDSR-hop) predicts MDD as does the rumination subscale 
(LEIDS-R-rum). Finally, the LEIDS-R-aggression is a predictor for DD.

The omnibus binomial logistic regression model indicated a significant model overall for each of the disorders (see Tables 3,4) as well as a reasonable proportion of explained variance (Nagelkerke $R^{2}$-varied from 0.12 to 0.18). Considering the type of analysis-using a built in psychiatric control group and thus exclusion of healthy controls - these figures indicate that a reasonable proportion of the variance can be explained by each of the models.

When comparing these final models to the versions without the cognitive constructs, - only including demographics and the personality traits of neuroticism and extraversion-, all models significantly improved their fit as a result of adding the cognitive constructs. For SAD the improvement was $\chi^{2}(2, N=462)=28.20, P<0.001$, for GAD $\chi^{2}(1, N=319)=33.93, P<0.001$, for PD $\chi^{2}(1$, $N=419)=124.60, P<0.001$, for DD: $\chi^{2}(1, N=226)=$ 11.85, $P=0.001$ and for MDD: $\chi^{2}(3, N=644)=57.95$, $P<0.001$.

\section{Prediction of Disorders in Remission by Cognitive Constructs After Correction for Personality Traits}

In contrast to the previous models that aimed to predict current diagnosis, the next models aim to investigate whether the established relationships will hold up when disorders are in remission without current diagnoses being able to dominate the relationship. In order to do so all participants with no history of depressive or anxiety disorders and all participants currently suffering from a disorder were excluded from the analyses, resulting in a sample of $N=834$. Moreover, in order to correct for residual symptoms the covariates BAI and IDS where added to the models. Results are presented in Tables 5 and 6. These tables also show model fit and explained variance (Nagelkerke $R^{2}$ ) for each model.

Results for the anxiety disorders were very similar to what was reported for current diagnosis: ASI-social-cognitive concerns predicted SAD and ASI-physical concerns PD. Surprisingly PSWQ was the only exception as its contribution became non-significant in the prediction model of GAD where its influence seems to be completely overruled by neuroticism which now is a highly significant predictor.

Results for the depressive disorders show LEIDS-R-agg as predictor for DD and LEIDS-R-rum as a predictor of MDD. Most notable is the non-significant contribution of LEIDS-R-hop, which did add significantly to MDD's current disorder model.

All models are significant overall (see Tables 5, 6) although the explained variance is slightly lower than for the current disorders. Nagelkerke varied from 0.04 to 0.13 with PD scoring at the higher and GAD at the lower end.

When comparing these final models to the versions without the cognitive constructs-only including demographics, the BAI and IDS, and the personality traits of neuroticism and extraversion-all models, except for GAD where neuroticism dominated, significantly improved their fit as a result of adding the cognitive constructs. For SAD the improvement is $\chi^{2}(2, N=192)=21.20, P<0.001$, for PD $\chi^{2}(2, N=187)=42.13, P<0.001$, DD $\chi^{2}(1, N=$ $157)=9.20, P<0.01$ and for $\operatorname{MDD} \chi^{2}(2, N=693)=$ 20.30, $P<0.001$.

\section{Repeating Analyses with Comorbidity Correction}

As comorbidity among anxiety and depressive disorders is very common this could have influenced the results. In order to check this, analyses for both the current and remission sample were re-run, controlling for comorbidity by adding to each model the different diagnoses (dummy coded variables: absent/present) as covariates (data not shown). Hence, in each sample five binomial logistic regression analyses were run. The models consisted of demographics, personality traits and the different diagnoses with the exception of the index disorder (forced entry), and the cognitive variables (stepwise backward procedure). The same was done for the remission sample with the addition of BAI and IDS (forced entry) to control for residual symptoms.

Compared to the analyses without comorbidity correction, results in the current diagnosis sample showed only a minor difference in the model of DD: neuroticism no longer made a significant contribution. The models on disorders in remission showed no differences. Overall the results are very similar to the analyses without correction for comorbidity, with the same significant cognitive predictors still present in the models, indicating that the reported results are very robust and not critically confounded by comorbidity.

\section{Discussion}

The aim of this study was to investigate the incremental validity of the cognitive constructs $\mathrm{AS}, \mathrm{PW}$ and $\mathrm{CR}$ in predicting depressive and anxiety disorders over and above the personality traits of neuroticism and extraversion, while controlling for sociodemographic characteristics. This was investigated in both symptomatic and remitted patients.

Results from the symptomatic group revealed that both specific and unique cognitive components exist: Anxiety Sensitivity is a specific component involved in both SAD and PD, and Pathological Worry a unique component for 
GAD. Within the depressive disorders, Aggression Reactivity (LEIDS-R-agg) is unique for DD, and Rumination on Sadness (LEIDS-R-rum) and Hopelessness Reactivity (LEIDS-R-hop) are unique factors in MDD. As expected, odds ratios were small, but when considering that we applied a stringent test by using a psychiatric control group, these results are noteworthy and robust.

The subscales of AS have unique aspects; social-cognitive concerns (ASI-scc) is solely related to SAD and physical concerns (ASI-phc) to PD. At a higher-order level both factors capture the same underlying mechanism - the fear of anxiety related sensations. The involvement of AS in both PD and SAD was no surprise, as misinterpretation of bodily sensations and maladaptive cognitions such as the fear to lose control are well known to play a prominent role in these disorders (e.g., Clark 1988; Clark and Wells 1995).

PW is also a unique component. Note that 'uniqueness' does not mean that the construct is not involved in other disorders, but that it has a dominant role in one disorder. As pointed out by Mineka et al. (1998) "symptom specificity must be viewed in relative rather than absolute terms.". So even though PW is elevated in many disorders, it has a more defining role in GAD, which is in line with both cognitive models and the DSM classification (APA 2000). Two other unique components are Rumination on Sadness and Hopelessness Reactivity, which are both linked to MDD. This relationship is unique in the sense that their predictive value of MDD is over and above that of personality traits and anxiety constructs, and remains limited to this one disorder. Further, this finding is consistent with previous research highlighting the core role of rumination and hopelessness in maintaining and predicting depression (e.g., Alloy et al. 1999; Nolen-Hoeksema 2000).

Contrary to the above findings, the relationship between DD and Aggression Reactivity (LEIDS-R-agg) was somewhat unexpected. Irritability is a common symptom of DD but has not been investigated as a (cognitive) vulnerability factor. For example, Fava et al. (1997) reported increased anger attacks among people with dysthymia or atypical depression compared to normal controls. More recently, irritability was examined as a potential subtype of MDD in the general population and it was found that the presence of irritability (vs. its absence) in MDD is associated with higher comorbid dysthymia and lifetime persistence of symptoms (Fava et al. 2010). Further, a recent large-scale longitudinal study showed that irritability during adolescence predicts an adult diagnosis of dysthymia, and to a lesser extent GAD and MDD, over a period of 20 years (Stringaris et al. 2009). The present finding expands on this literature by showing a unique association between aggression reactivity and dysthymia, but not major depression. It is noteworthy that dysthymia was also related to lower extraversion in our sample. At first glance this pattern of results is suggestive of an introvert profile characterizing the dysthymic patient consistent with older psychoanalytic theories focusing on the central role of anger in depressive disorders. In this orientation difficulties with the expression of anger are thought to cause intrapsychic conflicts that lead to anger being directed inwards (Busch 2009). As a result, defence mechanisms, such as passive aggression, are triggered and maintained in dysthymic patients (Bloch et al. 1993). It should be noted however, that aggression reactivity is conscious, selfreported aggression.

The findings found in acute patients also appeared in the remission group, and the similarities are striking. With the exception of LEIDS-R-hop and PW all cognitive constructs uphold their position in the prediction models. An explanation for the non-significant contribution of LEIDS-R-hop to MDD can be found in a recently conducted study. This study showed that only remitted depressed participants with a history of suicidal ideation during their prior depressive episode are likely to experience high hopelessness reactivity scores during remission, whereas participants without such history do not show elevated hopelessness (Antypa et al. 2010). Consequently, the level of LEIDS-R-hop during remission seems to be dependent upon prior suicidal ideation status. This implies that although hopelessness was not represented in the overall MDD remission model it could still be a unique component for a specific subgroup. Another cognitive predictor conspicuous by its absence is PW. In the remission model of GAD, PW was no longer a significant predictor. This is most likely due to the high shared variance with neuroticism (e.g., Wells 1994). The cognitive predictors that continued to make a significant contribution to the remission models are AS (specific component: SAD and PD), LEIDS-R-agg (unique component: DD) and LEIDS-R-rum (unique component: MDD). The fact that these constructs uphold their position in the remission models, even when corrected for current symptomatology, supports the idea that these constructs are not merely epiphenomena of current disorders. Whether the results reflect scarring or more stable vulnerability factors cannot be derived from the present data.

The present study has several strengths such as the large (clinical) sample size, the recruitment from diverse settings, the replication in a remission group and the inclusion of several affective disorders and cognitive constructs.

There are also some limitations. The cross-sectional design limits insight into the direction of relationships. This was partly circumvented by also investigating remitted patients, however longitudinal data are needed to investigate cause and effect relationships. Another limitation lies in the selection of cognitive constructs. There are many other cognitive constructs which would have been 
interesting to include, such as intolerance of uncertainty, and experiential avoidance. Thirdly, the self-report nature of some of the instruments used in the present study has obvious downsides. Most of these measures are well established and accepted in research, however. The only exception is the LEIDS-R, which has a relatively short history. Although the present golden standard for CR measurement involves a mood induction (Scher et al. 2005), this has also its drawbacks and the success rates of inducing sadness also varies. Furthermore, as mentioned above, the support for the LEIDS-R as a valid measure of CR is accumulating (e.g., Moulds et al. 2008). Finally, the categorical diagnosis/no-diagnosis approach is necessarily accompanied by the issue of subsyndromal symptoms. In the remission sample the BAI and IDS were added as control variables in order to correct for residual symptoms. These measures however, do not cover the entire symptomatology spectrum of anxiety in particular and thus the presence of some residual (anxiety) symptoms cannot be excluded. The current diagnosis sample did not allow for a similar approach to correct for subsyndromal (comorbid) symptoms as this would have corrected for the severity of the current index disorder itself and hence analyses would have provided non-informative results. Therefore it cannot be excluded that subsyndromal comorbid symptoms might account for some of the associations of current disorders with global or specific cognitive factors. Overall the findings support the Integrative Hierarchical Model. Moreover, when considering the additional value of cognitive constructs in understanding anxiety and depressive disorders, one can conclude that reliance on a few general measures does not do justice to the complexity of these psychopathologies. On a more practical level the results imply that although a patient might no longer meet (symptomatic) criteria of a disorder, cognitive mind sets might still be latently present, increasing the risk for relapse.

\begin{abstract}
Acknowledgments The infrastructure for the NESDA study (www.nesda.nl) is funded through the Geestkracht program of the Netherlands Organisation for Health Research and Development (Zon-Mw, grant number 10-000-1002) and is supported by participating universities and mental health care organizations (VU University Medical Center, GGZ inGeest, Arkin, Leiden University Medical Center, GGZ Rivierduinen, University Medical Center Groningen, Lentis, GGZ Friesland, GGZ Drenthe, Scientific Institute for Quality of Healthcare (IQ healthcare), Netherlands Institute for Health Services Research (NIVEL) and Netherlands Institute of Mental Health and Addiction (Trimbos Institute). A. J. W. Van der Does is supported by Netherlands Organisation for Scientific Research (NWO) Vici Grant \# 453-06-005.
\end{abstract}

Open Access This article is distributed under the terms of the Creative Commons Attribution Noncommercial License which permits any noncommercial use, distribution, and reproduction in any medium, provided the original author(s) and source are credited.

\section{References}

Alloy, L. B., Abramson, L. Y., Whitehouse, W. G., Hogan, M. E., Tashman, N. A., Steinberg, D. L., et al. (1999). Depressogenic cognitive styles: Predictive validity, information processing and personality characteristics, and developmental origins. Behaviour Research and Therapy, 37(6), 503-531.

Angst, J. (1996). Comorbidity of mood disorders: A longitudinal prospective study. British Journal of Psychiatry, 168, 31-37.

Antypa, N., Van der Does, A. J. W., \& Penninx, B. W. J. H. (2010). Cognitive reactivity: Investigation of a potentially treatable marker of suicide risk in depression. Journal of Affective Disorders, 122(1-2), 46-52.

APA. (1994). Diagnostic and statistical manual of mental disorders (4th ed.). Washington, DC: Author.

APA. (2000). Diagnostic and statistical manual of mental disorders (4th ed.). Washington, DC: Author.

Beck, A. T., Brown, G., Epstein, N., \& Steer, R. A. (1988). An inventory for measuring clinical anxiety-Psychometric properties. Journal of Consulting and Clinical Psychology, 56(6), 893-897.

Bijl, R. V., van Zessen, G., Ravelli, A., de Rijk, C., \& Langendoen, Y. (1998). The Netherlands mental health survey and incidence study (NEMESIS): Objectives and design. Social Psychiatry and Psychiatric Epidemiology, 33(12), 581-586.

Bloch, A. L., Shear, M. K., Markowitz, J. C., Leon, A. C., \& Perry, J. C. (1993). An empirical study of defense mechanisms in dysthymia. American Journal of Psychiatry, 150(8), 1194-1198.

Booij, L., \& Van der Does, A. J. W. (2007). Cognitive and serotonergic vulnerability to depression: Convergent findings. Journal of Abnormal Psychology, 116(1), 86-94.

Borkovec, T. D. (1994). The nature, functions and origins of worry. In G. C. L. Davey \& F. Tallis (Eds.), Worrying: Perspectives on theory, assessment and treatment. Chichester, England: Wiley.

Brown, T. A., Antony, M. M., \& Barlow, D. H. (1992). Psychometric properties of the Penn State Worry Questionnaire in a clinical anxiety disorders sample. Behaviour Research and Therapy, 30(1), 33-37.

Brown, T. A., Chorpita, B. F., \& Barlow, D. H. (1998). Structural relationships among dimensions of the DSM-IV anxiety and mood disorders and dimensions of negative affect, positive affect, and autonomic arousal. Journal of Abnormal Psychology, 107(2), 179-192.

Busch, F. N. (2009). Anger and depression. Advances in Psychiatric Treatment, 15(4), 271-278.

Chelminski, I., \& Zimmerman, M. (2003). Pathological worry in depressed and anxious patients. Journal of Anxiety Disorders, 17(5), 533-546.

Clark, D. M. (1988). A cognitive model of panic attacks. In S. Rachman \& J. D. Maser (Eds.), Panic: Psychological perspectives (pp. 71-89). Hillsdale, NJ: Erlbaum.

Clark, L. A., \& Watson, D. (1991). Tripartite model of anxiety and depression-Psychometric evidence and taxonomic implications. Journal of Abnormal Psychology, 100(3), 316-336.

Clark, D. M., \& Wells, A. (1995). A cognitive model of social phobia. In R. G. Heimberg, M. R. Liebowitz, D. A. Hope, \& F. R. Schneier (Eds.), Social phobia: Diagnosis, assessment and treatment. New York: Guilford Press.

Costa, P. T., \& Mccrae, R. R. (1995). Domains and facetsHierarchical personality-assessment using the revised neo personality-inventory. Journal of Personality Assessment, 64(1), 21-50.

Cox, B. J., Borger, S. C., Taylor, S., Fuentes, K., \& Ross, L. M. (1999). Anxiety sensitivity and the five-factor model of personality. Behaviour Research and Therapy, 37(7), 633-641. 
Cox, B. J., Parker, J. D. A., \& Swinson, R. P. (1996). Anxiety sensitivity: Confirmatory evidence for a multidimensional construct. Behaviour Research and Therapy, 34(7), 591-598.

Davey, G. C. L. (1993). A comparison of 3 worry questionnaires. Behaviour Research and Therapy, 31(1), 51-56.

de Graaf, R., Bijl, R. V., Smit, F., Vollebergh, W. A., \& Spijker, J. (2002). Risk factors for 12-month comorbidity of mood, anxiety, and substance use disorders: Findings from the Netherlands Mental Health Survey and Incidence Study. American Journal of Psychiatry, 159(4), 620-629.

Farmer, A. E., Katz, R., Mcguffin, P., \& Bebbington, P. (1987). A comparison between the present state examination and the composite international diagnostic interview. Archives of General Psychiatry, 44(12), 1064-1068.

Fava, M., Hwang, I., Rush, A. J., Sampson, N., Walters, E. E., \& Kessler, R. C. (2010). The importance of irritability as a symptom of major depressive disorder: Results from the National Comorbidity Survey Replication. Molecular Psychiatry, $15,856-867$.

Fava, M., Nierenberg, A. A., Quitkin, F. M., Zisook, S., Pearlstein, T., Stone, A., et al. (1997). A preliminary study on the efficacy of sertraline and imipramine on anger attacks in atypical depression and dysthymia. Psychopharmacology Bulletin, 33(1), 101-103.

Ferguson, R. J. (2000). Using the Beck Anxiety Inventory in primary care. In M. E. Maruish (Ed.), Handbook of psychological assessment in primary care settings (pp. 509-534). Mahwah, NJ: Lawrence Erlbaum.

Field, A. (2005). Discovering statistics using SPSS (2nd ed.). London: SAGE.

Firk, C., \& Markus, C. R. (2009). Mood and cortisol responses following tryptophan-rich hydrolyzed protein and acute stress in healthy subjects with high and low cognitive reactivity to depression. Clinical Nutrition, 28(3), 266-271.

Gibb, B. E., Chelminski, I., \& Zimmerman, M. (2007). Childhood emotional, physical, and sexual abuse, and diagnoses of depressive and anxiety disorders in adult psychiatric outpatients. Depression and Anxiety, 24(4), 256-263.

Ingram, R. E. (1990). Self-focused attention in clinical disordersReview and a conceptual-model. Psychological Bulletin, 107(2), 156-176.

Kessler, R. C., McGonagle, K. A., Zhao, S., Nelson, C. B., Hughes, M., Eshleman, S., et al. (1994). Lifetime and 12-month prevalence of DSM-III-R psychiatric disorders in the United States. Results from the national comorbidity survey. Archives of General Psychiatry, 51(1), 8-19.

Kotov, R., Watson, D., Robles, J. P., \& Schmidt, N. B. (2007). Personality traits and anxiety symptoms: The multilevel trait predictor model. Behaviour Research and Therapy, 45(7), $1485-1503$.

Landman-Peeters, K. M. C., Hartman, C. A., van der Pompe, G., den Boer, J. A., Minderaa, R. B., \& Ormel, J. (2005). Gender differences in the relation between social support, problems in parent-offspring communication, and depression and anxiety. Social Science and Medicine, 60(11), 2549-2559.

Menard, S. (1995). Applied logistic regression analysis (Vol. 07-106). Thousand Oaks, CA: Sage.

Merens, W., Booij, L., Markus, R., Zitman, F. G., Onkenhout, W., \& Van der Does, A. J. (2005). The effects of a diet enriched with alpha-lactalbumin on mood and cortisol response in unmedicated recovered depressed subjects and controls. British Journal of Nutrition, 94(3), 415-422.

Meyer, T. J., Miller, M. L., Metzger, R. L., \& Borkovec, T. D. (1990). Development and validation of the Penn State Worry Questionnaire. Behaviour Research and Therapy, 28(6), 487-495.
Mineka, S., Watson, D., \& Clark, L. A. (1998). Comorbidity of anxiety and unipolar mood disorders. Annual Review of Psychology, 49, 377-412.

Miranda, J., Gross, J. J., Persons, J. B., \& Hahn, J. (1998). Mood matters: Negative mood induction activates dysfunctional attitudes in women vulnerable to depression. Cognitive Therapy and Research, 22(4), 363-376.

Molina, S., \& Borkovec, T. D. (1994). The Penn State Worry Questionnaire: Psychometric properties and associated characteristics. In G. C. L. Davey \& F. Tallis (Eds.), Worrying perspectives on theory, assessment and treatment. Chichester: Wiley.

Moulds, M. L., Kandris, E., Williams, A. D., Lang, T., Yap, C., \& Hoffmeister, K. (2008). An investigation of the relationship between cognitive reactivity and rumination. Behavior Therapy, 39(1), 65-71.

Myers, R. (1990). Classical and modern regression with applications (2nd ed.). Boston, MA: Duxbury.

Nolen-Hoeksema, S. (2000). The role of rumination in depressive disorders and mixed anxiety/depressive symptoms. Journal of Abnormal Psychology, 109(3), 504-511.

Norton, P. J., \& Mehta, P. D. (2007). Hierarchical model of vulnerabilities for emotional disorders. Cognitive Behaviour Therapy, 36(4), 240-254.

Norton, P. J., Sexton, K. A., Walker, J. R., \& Norton, G. R. (2005). Hierarchical model of vulnerabilities for anxiety: Replication and extension with a clinical sample. Cognitive Behaviour Therapy, 34(1), 50-63.

Otto, M. W., Pollack, M. H., Fava, M., Uccello, R., \& Rosenbaum, J. F. (1995). Elevated anxiety sensitivity index scores in patients with major depression-Correlates and changes with antidepressant treatment. Journal of Anxiety Disorders, 9(2), 117-123.

Penninx, B. W. J. H., Beekman, A. T. F., Smit, J. H., Zitman, F. G., Nolen, W. A., Spinhoven, P., et al. (2008). The Netherlands study of depression and anxiety (NESDA): rationale, objectives and methods. International Journal of Methods in Psychiatric Research, 17(3), 121-140.

Peterson, R. A., \& Plehn, K. (1999). Measuring anxiety sensitivity. In S. Taylor (Ed.), Anxiety sensitivity: Theory, research, and treatment of the fear of anxiety (pp. 61-81). Mahwah, NJ: Erlbaum.

Peterson, R. A., \& Reiss, S. (1992). Anxiety sensitivity index revised test manual. Worthington, $\mathrm{OH}$ : International Diagnostic Services.

Prenoveau, J. M., Zinbarg, R. E., Craske, M. G., Mineka, S., Griffith, J. W., \& Epstein, A. M. (2010). Testing a hierarchical model of anxiety and depression in adolescents: A tri-level model. Journal of Anxiety Disorders, 24(3), 334-344.

Rector, N. A., Szacun-Shimizu, K., \& Leybman, M. (2007). Anxiety sensitivity within the anxiety disorders: Disorder-specific sensitivities and depression comorbidity. Behaviour Research and Therapy, 45(8), 1967-1975.

Reiss, S., \& McNally, R. J. (1985). Expectancy model of fear. In S. Reiss \& R. R. Bootzin (Eds.), Theoretical issues in behavior therapy (pp. 107-121). San Diego, CA: Academic Press.

Reiss, S., Peterson, R. A., Gursky, D. M., \& Mcnally, R. J. (1986). Anxiety sensitivity, anxiety frequency and the prediction of fearfulness. Behaviour Research and Therapy, 24(1), 1-8.

Rodriguez, B. F., Bruce, S. E., Pagano, M. E., Spencer, M. A., \& Keller, M. B. (2004). Factor structure and stability of the Anxiety Sensitivity Index in a longitudinal study of anxiety disorder patients. Behaviour Research and Therapy, 42(1), 79-91.

Rush, A. J., Gullion, C. M., Basco, M. R., Jarrett, R. B., \& Trivedi, M H. (1996). The inventory of depressive symptomatology (IDS): 
Psychometric properties. Psychological Medicine, 26(3), 477486.

Sartorius, N., Ustun, T. B., Lecrubier, Y., \& Wittchen, H. U. (1996). Depression comorbid with anxiety: results from the WHO study on psychological disorders in primary health care. British Journal of Psychiatry Supplement, 168(Suppl. 30), 38-43.

Scher, C. D., Ingram, R. E., \& Segal, Z. V. (2005). Cognitive reactivity and vulnerability: Empirical evaluation of construct activation and cognitive diatheses in unipolar depression. Clinical Psychology Review, 25(4), 487-510.

Schmidt, N. B., \& Joiner, T. E. (2002). Structure of the Anxiety Sensitivity Index psychometrics and factor structure in a community sample. Journal of Anxiety Disorders, 16(1), 33-49.

Segal, Z. V., Gemar, M., \& Williams, S. (1999). Differential cognitive response to a mood challenge following successful cognitive therapy or pharmacotherapy for unipolar depression. Journal of Abnormal Psychology, 108(1), 3-10.

Segal, Z. V., Kennedy, S., Gemar, M., Hood, K., Pedersen, R., \& Buis, T. (2006). Cognitive reactivity to sad mood provocation and the prediction of depressive relapse. Archives of General Psychiatry, 63(7), 749-755.

Sexton, K. A., Norton, P. J., Walker, J. R., \& Norton, G. R. (2003). Hierarchical model of generalized and specific vulnerabilities in anxiety. Cognitive Behaviour Therapy, 32(2), 82-94.

Starcevic, V. (1995). Pathological worry in major depression-A preliminary-report. Behaviour Research and Therapy, 33(1), $55-56$.

Starcevic, V., Berle, D., Milicevic, D., Hannan, A., Lamplugh, C., \& Eslick, G. D. (2007). Pathological worry, anxiety disorders and the impact of co-occurrence with depressive and other anxiety disorders. Journal of Anxiety Disorders, 21(8), 10161027.

Stringaris, A., Cohen, P., Pine, D. S., \& Leibenluft, E. (2009). Adult outcomes of youth irritability: A 20-year prospective community-based study. American Journal of Psychiatry, 166(9), 1048-1054.

Ter Smitten, M. H., Smeets, R. M. W., \& Van den Brink, W. (1998). Composite international diagnostic interview (CIDI), version 2.1, 12 months [in Dutch]. Amsterdam: World Health Organization.

Van der Does, W. (2002). Cognitive reactivity to sad mood: structure and validity of a new measure. Behaviour Research and Therapy, 40(1), 105-120.
Van der Does, W. (2005). Thought suppression and cognitive vulnerability to depression. British Journal of Clinical Psychology, 44(Pt 1), 1-14.

van Rijsoort, S., Emmelkamp, P., \& Vervaeke, G. (1999). The Penn State Worry Questionnaire and the Worry Domains Questionnaire: Structure, reliability and validity. Clinical Psychology \& Psychotherapy, 6(4), 297-307.

Vujanovic, A. A., Arrindell, W. A., Bernstein, A., Norton, P. J., \& Zvolensky, M. J. (2007). Sixteen-item anxiety sensitivity index-Confirmatory factor analytic evidence, internal consistency, and construct validity in a young adult sample from the Netherlands. Assessment, 14(2), 129-143.

Wacker, H. R., Battegay, R., Mullejans, R., \& Schlosser, C. (2006). Using the CIDI-C in the general population. In C. N. Stefanis, A. D. Rabavilas, \& C. R. Soldatos (Eds.), Psychiatry: A world perspective (pp. 138-143). Amsterdam: Elsevier.

Watkins, E. R. (2008). Constructive and unconstructive repetitive thought. Psychological Bulletin, 134(2), 163-206.

Wells, A. (1994). A multidimensional measure of worry-Development and preliminary validation of the anxious thoughts inventory. Anxiety Stress and Coping, 6(4), 289-299.

Williams, J. M. G., Van der Does, A. J. W., Barnhofer, T., Crane, C., \& Segal, Z. S. (2008). Cognitive reactivity, suicidal ideation and future fluency: Preliminary investigation of a differential activation theory of hopelessness/suicidality. Cognitive Therapy and Research, 32(1), 83-104.

Wittchen, H. U. (1994). Reliability and validity studies of the who composite international diagnostic interview (Cidi)—A criticalreview. Journal of Psychiatric Research, 28(1), 57-84.

Wittchen, H. U., Burke, J. D., Semler, G., Pfister, H., Voncranach, M., \& Zaudig, M. (1989). Recall and dating of psychiatricsymptoms-Test-retest reliability of time-related symptom questions in a standardized psychiatric interview. Archives of General Psychiatry, 46(5), 437-443.

Wittchen, H. U., Robins, L. N., Cottler, L. B., Sartorius, N., Burke, J. D., \& Regier, D. (1991). Cross-cultural feasibility, reliability and sources of variance of the composite international diagnostic interview (Cidi). British Journal of Psychiatry, 159, 645-653.

Zinbarg, R. E., Barlow, D. H., \& Brown, T. A. (1997). Hierarchical structure and general factor saturation of the anxiety sensitivity index: Evidence and implications. Psychological Assessment, 9(3), 277-284. 